\title{
EDUCAÇÃO FÍSICA E SAÚDE MENTAL: ATUAÇÃO PROFISSIONAL NOS CENTROS DE ATENÇÃO PSICOSSOCIAL
}

\author{
Tatiane Motta da Costa e Silva \\ Universidade Federal do Pampa, Uruguaiana, Rio Grande do Sul, Brasil \\ Franciele Machado dos Santos \\ Universidade Federal do Pampa, Uruguaiana, Rio Grande do Sul, Brasil \\ Raquel Cristina Braun da Silva \\ Universidade Federal do Pampa, Uruguaiana, Rio Grande do Sul, Brasil \\ Helter Luiz da Rosa Oliveira \\ Universidade Federal do Pampa, Uruguaiana, Rio Grande do Sul, Brasil \\ Phillip Vilanova Ilha \\ Universidade Federal do Pampa, Uruguaiana, Rio Grande do Sul, Brasil \\ Susane Graup \\ Universidade Federal do Pampa, Uruguaiana, Rio Grande do Sul, Brasil
}

\begin{abstract}
Resumo
O objetivo do estudo foi caracterizar o perfil e as atividades desenvolvidas pelos Profissionais de Educação Física (PEF) nos Centros de Atenção Psicossocial (CAPS) dos municípios que compõe a $10^{\text {a }}$ Coordenadoria Regional de Saúde - RS. A coleta de dados se deu por meio de um questionário composto de perguntas abertas e fechadas. A amostra foi composta por 6 PEF. Os resultados indicam que os PEF não receberam em sua graduação nenhuma formação relacionada à saúde mental. A atuação dos PEF envolve práticas variadas como atividades esportivas e recreativas, ministradas nas dependências dos CAPS e em espaços públicos. Diante do que é exposto, sugere-se a inclusão de discussões sobre a temática nos cursos de graduação e no investimento em capacitação para os profissionais da área.
\end{abstract}

Palavras-chave: Educação Física. Serviços de saúde mental. Prática profissional.

\section{Introdução}

A Política Brasileira de Atenção em Saúde Mental tem suas bases no movimento denominado Reforma Psiquiátrica, que surgiu a partir de manifestações e debates, nos diferentes setores da sociedade, em prol dos direitos civis e políticos do paciente psiquiátrico e por um tratamento mais humanizado e antimanicomial (BRASIL, 2005). Em suma, a empreitada da reforma psiquiátrica foi substituir uma psiquiatria centralizada no hospital por uma psiquiatria amparada em dispositivos diversificados, abertos e de natureza comunitária ou territorial (TENÓRIO, 2002).

Apenas em 2001, esses usuários obtiveram uma lei que regulamenta uma política de saúde mental. A Lei 10.216, de 6 de abril de 2001, ratificou as diretrizes básicas que 
constituem o Sistema Único de Saúde (SUS), garantindo aos usuários de serviços de saúde mental e aos que sofrem transtornos decorrentes do consumo de álcool e outras drogas a universalidade de acesso e direito à assistência, bem como a sua integralidade (DUARTE; KANTORSKI, 2011). Neste contexto, o cuidado aos usuários deve ser realizado em serviços substitutivos aos hospitais psiquiátricos, inserindo, assim, os Centros de Atenção Psicossocial (CAPS) como locais de cuidado e assistência (ABUHAB et al., 2005).

Segundo a Portaria GM 224/92, os CAPS são definidos como unidades que oferecem atendimento multiprofissional de cuidados intermediários entre o regime ambulatorial e a internação hospitalar a uma população adstrita definida. De maneira geral, eles se caracterizam pela utilização intensiva de um conjunto amplo e complexo de tecnologias terapêuticas e práticas psicossociais dirigidas para o tratamento das pessoas com transtornos mentais ou que fazem o uso abusivo de álcool e outras drogas (PIMENTA; ROMAGNOLI, 2008).

Os CAPS são diferentes conforme a especificidade de sua população-alvo. De acordo com a Portaria GM 336/02, podem ser CAPS I (municípios com população acima de 20 mil habitantes) e CAPS II (acima de 70 mil), destinados ao atendimento diário de adultos, em sua população de abrangência, com transtornos mentais severos e persistentes; CAPS III, destinado à mesma população, com funcionamento 24 horas durante sete dias da semana; CAPSi, destinado à população infantil e adolescente com transtornos mentais e persistentes, e CAPSad, para atendimento diário à população com transtornos decorrentes do uso abusivo de substâncias psicoativas, como álcool e outras drogas.

Os CAPS asseguram aos indivíduos com transtornos mentais e/ou com problemas devido ao uso abusivo de álcool e outras drogas o direito de serem assistidos em um sistema comunitário de saúde mental, que tem como foco a desinstitucionalização, a reabilitação psicossocial e a reinserção social (TENÓRIO, 2002; SEVERO; DIMENSTEIN, 2011). Devido a essa nova forma de pensar o atendimento a esses indivíduos, novas abordagens e estratégias foram elaboradas, dando espaço para que outros profissionais possam fazer parte da rotina de cuidados desses usuários, e abrindo um leque de opções a ser trabalhadas. Tem assim seu lugar, na atenção à saúde mental, o Profissional de Educação Física (PEF).

As possibilidades de inserção do PEF no contexto da saúde pública aumentaram muito nos últimos anos. Conforme consta na Política Nacional de Promoção da Saúde (BRASIL, 2006), o PEF no campo da saúde, em especial no âmbito do SUS, bem como os demais profissionais que atuam na área do movimento humano, tem atribuições nas ações na rede básica de saúde e na comunidade.

As ações propostas pelos PEF, além de servirem como desenvolvimento e estratégia para a prática de atividades corporais, são um importante estímulo para que novos hábitos sejam adotados pelos usuários (OLIVEIRA et al., 2011). Além disso, os saberes e práticas não somente técnicos desses profissionais proporcionam aos sujeitos uma prática mais acolhedora, fato que potencializa o cuidado e facilita uma abordagem integral, aumentando a qualidade do atendimento aos sujeitos (SARAIVA; CREMONESE, 2008).

Neste sentido, a Educação Física relacionada à saúde mental é uma das alternativas de cuidado que deve ser trabalhada na atual proposta de atendimento, buscando uma visão e uma atuação multiprofissionais para que os usuários dos serviços de saúde mental sejam vistos e tratados em sua integralidade (WACHS, 2008; MIRANDA; FREIRE; OLIVEIRA, 2011). No entanto, a literatura vem apontando a necessidade de pesquisas que tratem da qualificação dos profissionais no âmbito da saúde mental, visto que esses profissionais muitas vezes sentem-se despreparados para atuar nesse campo (TAVARES, 2006; SILVA; OLIVEIRA; KAMIMURA, 2014).

Diante deste cenário, faz-se necessário analisar a atual situação da Educação Física nos CAPS, a fim de traçar um perfil dos profissionais da área e de compreender como ocorre 
o seu processo de trabalho. A investigação da correlação entre qualidade do serviço prestado e a formação dos PEF envolvidos no cuidado ampliará a discussão de novas práticas de cuidado que venham a contribuir para a qualidade da assistência em saúde mental.

Sendo assim, o objetivo do presente estudo foi caracterizar o perfil e as atividades desenvolvidas pelos profissionais de Educação Física nos Centros de Atenção Psicossocial que compõem a $10^{a}$ Coordenadoria Regional de Saúde (10a CRS) do estado do Rio Grande do Sul, considerando aspectos de sua formação, do vínculo profissional e da prática profissional nos serviços de saúde mental.

\section{Métodos}

Trata-se de um estudo descritivo, qualitativo e quantitativo realizado com Profissionais de Educação Física (PEF), atuantes nos Centros de Atenção Psicossocial dos municípios que compõem a $10^{a}$ CRS do estado do Rio Grande do Sul. O presente estudo foi aprovado pelo Comitê de Ética em Pesquisa da instituição dos pesquisadores sob o número de parecer 1.762.471 e seguiu as orientações da Resolução no 466/12 do Conselho Nacional de Saúde.

A $10^{a}$ CRS cobre um território que abrange 11 municípios. Destes, 7 municípios possuem Centros de Atenção Psicossocial, dos quais 4 municípios têm mais de um CAPS, resultando num total de 12 instituições, distribuídas conforme o apresentado no Quadro 1. Das 12 instituições, apenas 7 CAPS contam com PEF, e apenas 6 destes profissionais participaram do estudo.

\section{Quadro 1 - Amostra dos municípios e seus respectivos CAPS}

\begin{tabular}{|c|c|c|c|}
\hline Município & \multicolumn{3}{|c|}{ Centro de Atenção Psicossocial } \\
\hline Alegrete & CAPS II & CAPSad & CAPSi \\
\hline Itaqui & CAPS I & CAPS I & \\
\hline Quaraí & CAPS I & & \\
\hline Rosário do Sul & CAPS I & & \\
\hline Santana do Livramento & CAPS I & CAPSad & \\
\hline São Gabriel & CAPS I & & \\
\hline Uruguaiana & CAPS II & CAPSad & \\
\hline
\end{tabular}

Fonte: Elaborado pelos autores (2016).

A amostra do estudo foi composta por 6 profissionais do sexo masculino, com idade entre 32 e 41 anos (Média= 36,5 DP= 5,61). A perda amostral se deu pelo fato de um dos sujeitos da pesquisa estar afastado das atividades de trabalho. Para garantir o anonimato dos sujeitos da pesquisa, eles serão identificados no estudo por letras de A a F.

$\mathrm{O}$ procedimento de coleta de dados foi realizado por meio de um questionário elaborado especificamente para o presente estudo, sendo este documento composto de perguntas abertas e fechadas. As questões buscaram identificar informações relativas aos indicadores de formação acadêmica, satisfação profissional, vínculo profissional, formação continuada, prática profissional diária e atividades desenvolvidas com os usuários. Antes de responder ao questionário, os participantes assinaram um termo de consentimento livre e esclarecido.

Para a realização da coleta de dados, foi realizada uma visita aos locais de trabalho dos profissionais e aplicado o questionário de acordo com a disponibilidade dos serviços e dos participantes. O período de coleta de dados foi de outubro a novembro de 2016.

Para analisar as questões abertas do questionário, utilizou-se, como principal aporte metodológico, a análise de conteúdo proposto por Bardin (2011), em que a análise de 
conteúdo foi fundamentada na análise categorial, com desmembramento das respostas em categorias, constituídas por temas que emergiam delas. As respostas passaram por um crivo de classificação, segundo a frequência de presença ou ausência de itens de sentido.

\section{Resultados e discussão}

O Quadro 2 apresenta os resultados encontrados quanto aos indicadores relacionados à formação acadêmica, sendo possível identificar que todos os profissionais concluíram sua graduação em universidade privada e relataram não ter recebido nenhuma formação sobre saúde mental na graduação. Metade realizou pós-graduação, todos em universidade pública. Este resultado precisa ser considerado, uma vez que as evidências científicas apontam que os PEF encontram dificuldades de inserção nos serviços de saúde mental, por não terem nenhum conhecimento sobre a temática nem sobre a atuação profissional nestes locais (MACHADO, 2015; FURTADO et al., 2015).

Quadro 2 - Indicadores relacionados à formação acadêmica dos PEF

\begin{tabular}{|lccccc|}
\hline & $\begin{array}{c}\text { Tipo de } \\
\text { Graduação }\end{array}$ & Universidade & $\begin{array}{c}\text { Pós- } \\
\text { graduação }\end{array}$ & Universidade & $\begin{array}{c}\text { Formação sobre } \\
\text { saúde mental na } \\
\text { graduação }\end{array}$ \\
\hline A & Licenciatura Plena & Privada & Especialização & Pública & Não recebeu \\
B & Licenciatura Plena & Privada & Não possui & - & Não recebeu \\
C & Licenciatura/ & Privada & Não possui & - & Não recebeu \\
& Bacharelado & & & & Não recebeu \\
D & Licenciatura & Privada & Especialização & Pública & Não recebeu \\
E & Licenciatura Plena & Privada & Especialização & Pública & Não recebeu \\
F & Licenciatura & Privada & Não possui & - & \\
\hline
\end{tabular}

Fonte: Elaborado pelos autores (2016).

Na perspectiva de qualificar os PEF para o trabalho em saúde mental, é necessário que se invista em sua formação durante o processo acadêmico. Machado (2015) afirma que as dificuldades de inserção dos PEF nos serviços de saúde mental poderiam ser reduzidas durante o período de formação acadêmica.

Mesmo após o seu reconhecimento como profissão da saúde, a Educação Física é uma das áreas da saúde na qual pouco se discute o SUS (MACHADO, 2007). Desta forma, enquanto as diretrizes de outros cursos da área destacam a importância de uma formação comprometida com o SUS, nas diretrizes da Educação Física ele fica marginalizado (WACHS, 2008).

As informações relacionadas ao vínculo profissional, a experiências anteriores com a área e ao motivo que levou os sujeitos a escolherem o trabalho em saúde mental são apresentadas no Quadro 3.

Quadro 3 - Características do trabalho dos PEF

\begin{tabular}{|ccccc|}
\hline $\begin{array}{l}\text { Tempo de } \\
\text { trabalho no }\end{array}$ & $\begin{array}{l}\text { Carga horária } \\
\text { semanal (horas) }\end{array}$ & Função no serviço & $\begin{array}{l}\text { Experiências } \\
\text { anteriores }\end{array}$ & $\begin{array}{l}\text { Motivos para o } \\
\text { trabalho em }\end{array}$ \\
\hline
\end{tabular}




\begin{tabular}{|lccccc|}
\hline & CAPS (anos) & & & saúde mental \\
\hline A & 12 & 40 & Coordenador & SIM & Afinidade \\
B & 1 & 20 & Professor de EF & NÃO & Indicação \\
C & 4 & 2 & Professor de EF & NÃO & Afinidade \\
D & 8 & 16 & Professor de EF & SIM & Afinidade \\
E & 9 & 20 & Coordenador & SIM & Indicação \\
F & 1 & 20 & Professor de EF & SIM & Indicação \\
\hline
\end{tabular}

Fonte: Elaborado pelos autores (2016).

Contatou-se que o tempo de trabalho dos PEF no CAPS variou de 1 ano a 12 anos (média $=5,83, \mathrm{DP}=4,53$ ). Em relação à carga horária semanal, ela varia de acordo com os serviços onde os PEF atuam, atingindo uma carga horária semanal entre 2 horas e 40 horas (média= 19,6, DP= 12,1).

Os sujeitos A, D, E e F apresentavam experiências anteriores em serviços como consultório na rua, ala terapêutica de hospital geral e outros CAPS, diferente dos demais, que não tinham experiências anteriores em serviços de atenção à saúde mental. Dados similares foram encontrados no estudo de Furtado et. al. (2015), no qual os autores constataram que os participantes, ao iniciarem o trabalho no CAPS, não tinham nenhuma familiaridade com o campo da saúde mental.

O trabalho no campo da saúde mental apresenta inúmeros desafios para a atuação do PEF, pois lidar com o sofrimento psíquico dos usuários é um limitador, principalmente no início de suas experiências, quando se deparam com uma realidade com a qual não estão acostumados e que não foi apresentada durante a formação (FURTADO et al., 2015). Os autores afirmam ainda que a inserção do profissional no serviço é permeada também por valores individuais, familiares, sociais, entre outros elementos.

Quanto à escolha para trabalhar na área de saúde mental, os sujeitos B, E e F responderam que não foi uma escolha e sim uma indicação, conforme demonstra a resposta do sujeito F: "[...] não foi uma escolha, fui chamado para trabalhar no CAPS e aceitei a oportunidade". Diferente dos professores A, C e D, que optaram por trabalhar no campo da saúde mental, o sujeito D justifica sua escolha: "[...] escolhi trabalhar no CAPS devido ao carinho e gratidão que recebo dos usuários".

Em relação à satisfação profissional, constatou-se que todos os participantes sentem-se satisfeitos com sua atuação profissional na saúde mental e relatam manter um relacionamento excelente com os usuários. Em relação aos demais membros da equipe profissional, os PEF indicaram ter um relacionamento muito bom (2) e excelente (4). A maioria dos PEF sente-se satisfeita com a remuneração mensal, e apenas dois profissionais apontaram como não satisfatório o salário mensal recebido.

Em relação às condições de trabalho, dois participantes apontaram como não satisfatório, indicando a falta de material como a principal queixa. "[...] o espaço físico é ótimo, porém, a falta de materiais acaba por nos limitar, tornando as condições de trabalho não tão satisfatórias assim" (extrato da resposta do profissional D).

Os demais profissionais relatam estar satisfeitos com as condições de trabalho e mencionam que desenvolvem as atividades práticas sem dificuldades, conforme consta na resposta do profissional C: "Normalmente consigo realizar minhas atividades com facilidade, raramente encontro alguma dificuldade, mas nada que atrapalhe o andamento das atividades".

A falta de material para a prática das atividades foi abordada na pesquisa de Machado (2015), na qual relata que esta também é uma dificuldade presente nos CAPS onde o estudo foi realizado e que essas situações fazem com que os profissionais tenham que pensar em outras possibilidades caso a estratégia inicial não possa ser concretizada. Fato semelhante foi constatado na pesquisa de Ferreira (2013), que relata a falta de recursos materiais para as oficinas terapêuticas dos CAPS, situação que considera recorrente em serviços do SUS, 
principalmente devido a processos licitatórios que impedem a chegada do material em tempo favorável.

Ressalta-se o bom relacionamento dos PEF com os demais profissionais da equipe e com os usuários como um importante fator que facilita o desenvolvimento das atividades multiprofissionais e fortalece o vínculo com os usuários, visto que a relação com os usuários pauta-se pela construção cotidiana do vínculo terapêutico. No trabalho em saúde, a construção do vínculo entre o profissional e o usuário é fundamental para que a relação seja de confiança e, sobretudo, mais humanizada (FURTADO et. al., 2015).

Os PEF têm maiores chances de se tornarem os terapeutas de referência dos usuários, isto porque estão envolvidos em algumas atividades que demandam maior tempo de convivência com o grupo, ao contrário de algumas oficinas que se limitam a alguns espaços/salas dentro do CAPS (MACHADO, 2015). Por desenvolverem atividades integrativas e que buscam o contato com o outro, o PEF cria uma aproximação com o usuário, refletindo positivamente no relacionamento entre profissional e usuário, como demonstra os resultados encontrados.

O Quadro 4 apresenta os dados relacionados à formação continuada e à capacitação para o trabalho na área de saúde mental, evidenciando que 4 CAPS realizam atividades de educação permanente e/ou continuada com os PEF. Essas atividades, segundo as respostas dos participantes, ocorrem durante as reuniões de equipes, conforme o extrato da resposta do sujeito E: "[...] as reuniões semanais de equipe, além das discussões de caso, servem como um espaço de educação permanente também”.

Quadro 4 - Indicadores relacionados à formação continuada dos PEF.

\begin{tabular}{|llcc|}
\hline & $\begin{array}{c}\text { Forma de procura } \\
\text { por formação } \\
\text { continuada }\end{array}$ & $\begin{array}{c}\text { Realização de Educação } \\
\text { Permanente e/ou } \\
\text { Continuada }\end{array}$ & $\begin{array}{c}\text { Capacitação inicial do } \\
\text { CAPS sobre saúde } \\
\text { mental }\end{array}$ \\
\hline A & Leituras & Realiza & Recebeu \\
B & Leituras e cursos & Não realiza & Não Recebeu \\
C & Leituras e cursos & Não realiza & Recebeu \\
D & Leituras, cursos & Realiza & Recebeu \\
& livros e revistas & Realiza & Recebeu \\
E & Leituras e cursos & Realiza & Recebeu \\
F & Leituras & &
\end{tabular}

Fonte: Elaborado pelos autores (2016).

As reuniões de equipe se apresentam como um espaço importante de formação e crescimento profissional, tanto individual quanto coletivo (MACHADO, 2015), além de serem caracterizadas como espaços potentes de discussão. A tomada de decisões por meio de reuniões de equipe e a pronta disponibilidade dos participantes de contribuírem para avaliação e intervenção nas diferentes situações que se apresentam no cotidiano de um serviço de saúde mental parecem ser uma estratégia para a superação das dificuldades educativas no âmbito da equipe (OLIVEIRA; SILVA, 2000; BABINSKI; HIRDES, 2004).

No entanto, é necessário ter cuidado para as discussões nas reuniões de equipe não serem baseadas apenas no conhecimento empírico e acabarem por reproduzir o que já vem sendo realizado. Estratégias de educação permanente são definidas por Tavares (2004) como programas amplos que visam articular conhecimento profissional específico com o de toda a rede de saberes envolvidos no sistema de saúde e devem substituir os programas de educação continuada, que, para a autora, são destinados apenas a informar os indivíduos sobre recentes avanços em seu campo de conhecimento, sem maiores discussões. 
A maioria dos PEF recebeu capacitação ao iniciar seu trabalho nos CAPS. Quando questionados sobre as contribuições destas ações para a prática profissional nos serviços de saúde mental, os sujeitos responderam que elas contribuíram para a abordagem, o acolhimento, o manejo de usuário em crise, a política de redução de danos, como demonstra o extrato da resposta do sujeito F - "[...] contribuiu, principalmente na forma de abordagem e acolhimento ao usuário" - e do sujeito E - “[...] ajudou na questão do manejo do usuário em crise e também a entender a política de redução de danos, que para mim foi uma novidade, pois não tinha nenhum conhecimento".

Quanto à importância de se qualificar e capacitar para trabalhar na área da saúde mental, todos os sujeitos concordam sobre essa necessidade, como podemos observar no extrato da resposta do sujeito E: “[...] importância de conhecer políticas públicas, áreas técnicas, manejo e questões que vêm a surgir; como não recebemos nenhuma formação na graduação, é preciso buscar esse conhecimento em outros espaços”. Fato também apresentado no Quadro 4, no qual os PEF indicam buscar leituras e a realização de cursos para se qualificar, evidenciando uma lacuna da formação.

A pesquisa de Silva, Oliveira e Kamimura (2014) realizou uma breve retrospectiva histórica sobre a capacitação em saúde mental, na qual constatou que a necessidade de capacitar as pessoas que trabalham ou apresentam perfil para trabalhar com saúde mental é reconhecida e reafirmada em todos os documentos publicados pelo Ministério da Saúde/SUS. Entretanto, a adequada formação técnica e teórica desses trabalhadores ainda é o grande desafio para a implantação do novo paradigma do cuidado na comunidade, demonstrando a necessidade de maiores investimentos em capacitações e cursos para o profissional.

O Quadro 5 expressa de modo sucinto as atividades realizadas pelos profissionais de Educação Física nos CAPS.

Quadro 5 - Atividades desenvolvidas com os usuários nos CAPS da região.

\begin{tabular}{|c|l|}
\hline Profissional & \multicolumn{1}{|c|}{ Atividades } \\
\hline A & Futebol, Futsal, Dança, Ginástica e Capoeira. \\
\hline B & $\begin{array}{l}\text { Futebol, Basquetebol, Voleibol, Bocha, Jogos de Mesa, Caminhada, Pescaria, } \\
\text { Academia ao ar livre. }\end{array}$ \\
\hline C & Aero Box, Circuito Funcional, Crox Fit e Caminhada. \\
\hline D & Futebol, Dança, Jogos Cooperativos e Trilha. \\
\hline E & Futebol, Caminhada, Alongamento, Cinema e Ginástica. \\
\hline F & $\begin{array}{l}\text { Futebol, Vôlei, Basquetebol, Tênis de Mesa, Caminhada, Academia ao ar livre e } \\
\text { Música. }\end{array}$ \\
\hline
\end{tabular}

Fonte: Elaborado pelos autores (2016).

Todos os profissionais relataram desenvolver suas atividades práticas nas dependências do CAPS e no território adstrito, em espaços públicos, como praças, parques, beira-rio, ginásio e academia ao ar livre. Conforme o relato dos PEF, as atividades são desenvolvidas em forma de grupos ou oficinas terapêuticas, semanalmente, com dias e horários definidos para cada atividade. Neste sentido, é necessário considerar essa prática como uma importante ação, pois, além de atenuar os problemas estruturais da instituição, contribui com o processo de desinstitucionalização e de reinserção social do usuário.

As atividades "extramuros" para usuários em tratamento no CAPS tornam-se um importante "acionador" do alcance ao bem-estar físico e mental, além da socialização deles com a realidade fora dos ambientes da instituição (MIRANDA; FREIRE; OLIVEIRA, 2011). 
Sendo assim, o estudo de Abib et al. (2010) também demonstra a possibilidade de aproximação dos espaços e do convívio com um público diverso da comunidade, constituindo a aproximação com a comunidade um dos principais objetivos dos CAPS.

Em relação às práticas ofertadas, é possível destacar algo simples como uma caminhada que envolve diversos benefícios a seus praticantes e é utilizada por quatro dos PEF participantes. Vale ressaltar que experiências como a caminhada possibilitam atenuar o estigma de doente que o usuário carrega e o qual a sociedade lhe impõe e ainda permitem que se diversifiquem o repertório das práticas de lazer (MACHADO, 2015). Também a experiência relatada por Cirqueira (2009) aponta que as atividades recreativas "despretensiosas" geram impactos positivos no cuidado e no processo de sociabilização.

Para Wachs (2008), é necessário que a Educação Física não seja imposta, mas que surja como demanda no próprio CAPS, possibilitando outras formas de organizar o cuidado na saúde mental. Através da prática dos mais diversos tipos de atividades desenvolvidas pelos profissionais de Educação Física, é possível influenciar positivamente na qualidade de vida dos usuários, uma vez que pesquisas direcionadas à qualidade de vida têm evidenciado cada vez mais a importância da atividade física e do exercício físico à saúde mental (WEINBERG; GOULD, 2001). A Educação Física é como uma ponte que liga aquele indivíduo isolado pela doença ao convívio com outras pessoas e, consequentemente, com a sociedade, desfrutando, assim, dos benefícios da sociabilidade (CORREIA, 2002).

As atividades esportivas também são citadas entre as práticas desenvolvidas pelos PEF, sendo o futebol a mais realizada pelos profissionais (5). A pesquisa de Abib et al. (2010) constatou que a oficina de futebol mobiliza uma série de elementos ligados à cultura corporal dos usuários do CAPS, especificamente, e do SUS, de modo geral. É uma, entre tantas ferramentas, que pode tornar possível a efetiva inserção das práticas corporais na saúde mental.

Essa prática pode vir a se tornar importante ferramenta terapêutica para o trabalho do CAPS, pois está intimamente ligada à cultura corporal local, podendo funcionar como dispositivo de mobilização de interesses dos usuários a ponto de potencializar o seu envolvimento nas comunidades onde vivem, contribuindo para a reinserção social desses sujeitos (ABIB et al., 2010).

Quando questionados se trabalham em conjunto com outro profissional, os sujeitos A, $\mathrm{B}, \mathrm{D}, \mathrm{E}$ e $\mathrm{F}$ descrevem que realizam suas atividades de forma multiprofissional, geralmente acompanhados por outros profissionais, como terapeuta ocupacional, psicólogo, assistente social, enfermeiro, entre outros. É mencionado também que a forma como ocorre esse trabalho multiprofissional se dá através da inserção dos colegas de trabalho nas atividades desenvolvidas pelos PEF.

Ceccim e Bilibio (2007) afirmam que a Educação Física pode contribuir para a efetivação de um novo campo de atuação e de outro tipo de prática que envolve o processo de saúde e doença e a atenção integral das pessoas em tratamento. A atuação dos PEF nesses espaços suscita algo além, pois possibilita um maior envolvimento com os usuários desses serviços. Por isso, a interação entre profissional, usuários e equipe multiprofissional mostra-se de suma importância para o trabalho significativo nos serviços de saúde mental (MACHADO, 2015).

Relativamente sobre a participação no Plano Terapêutico Singular dos usuários, os sujeitos B, C e F descreveram não participar da sua elaboração. Já os sujeitos A, D e E relatam que o plano terapêutico singular é discutido na reunião de equipe e posteriormente com o usuário, contando com a participação de vários profissionais envolvidos, conforme demonstra a resposta do sujeito D: "discute na reunião de equipe o plano terapêutico, depois, no momento do atendimento, se discute junto com o usuário e o familiar". 
Diante deste resultado, é possível perceber o quanto o trabalho de cada profissional se difere dos demais. Três profissionais relatam participar mais ativamente do tratamento do usuário e, quanto aos outros, a atuação do profissional fica mais restrita à parte prática da profissão, limitando-se a atividades práticas sem um maior envolvimento com o processo de tratamento do usuário nesses serviços.

A participação apenas de alguns PEF pode estar associada à forma com que os serviços se organizam, atribuindo a tarefa de construir o Projeto Terapêutico Singular dos usuários para um determinado membro da equipe multiprofissional, como demonstra a resposta do sujeito B: "[...] o projeto terapêutico singular é realizado pela pedagoga do CAPS". A discussão, portanto, fica limitada a esse profissional e não envolve os demais membros da equipe, o usuário e seus familiares. Além disso, o fato de os sujeitos $\mathrm{A}$ e $\mathrm{E}$ atuarem como coordenadores dos serviços pode interferir na forma como o profissional vai participar das decisões e na maneira como as atividades vão ser conduzidas, principalmente no que se refere à Educação Física.

Conforme a Portaria GM 336/02, o Projeto Terapêutico Sngular construído individualmente para cada usuário deve respeitar as diferenças regionais, as contribuições técnicas dos integrantes de sua equipe, as iniciativas locais dos familiares e do usuário e as articulações intersetoriais que potencializem suas ações. Assim, os trabalhadores devem participar coletivamente das decisões que são tomadas quanto ao tratamento dos usuários e ao andamento do serviço.

Quanto à percepção dos demais membros da equipe multiprofissional em relação à Educação Física, as respostas dos PEF se combinam, ressaltando a resposta do participante A: "[...] a Educação Física conquistou o seu espaço no CAPS, inclusive o psiquiatra reconhece os benefícios da atividade física e recomenda para os usuários". Na percepção dos sujeitos quanto à forma que a Educação Física é vista pelos usuários, os sujeitos novamente concordam, ressaltando a resposta do sujeito D: “[...] é vista como atrativo e fundamental, é a parte lúdica do plano terapêutico".

A partir do momento em que a Educação Física é valorizada pelos usuários, ela ganha reconhecimento e respeito diante da equipe de trabalho. Como mostra a pesquisa de Menezes (2010), que relata a aproximação com integrantes do CAPS infanto-juvenil a partir da caminhada, evidenciando que esses momentos são importantes, pois os usuários relatam situações de seu cotidiano, culminando na criação de vínculo e podendo contribuir ainda mais com o tratamento do usuário.

De modo semelhante, Santos et al. (2011), ao analisarem o trabalho da equipe de um CAPS na perspectiva das pessoas atendidas, chamam a atenção para as relações entre usuários e trabalhadores, afirmando que o profissional também precisa de preparo técnico-científico para criar vínculo com o usuário e para o bom exercício dessa inter-relação, pois o profissional de saúde deve demonstrar comprometimento com a realidade do usuário.

Para finalizar, foi questionado aos participantes o que mudariam na prática profissional. O sujeito A relatou que nada mudaria; já os sujeitos B, C, D e F relataram que buscariam mais conhecimento sobre a temática da saúde mental, por meio da realização de cursos, de capacitação e de pós-graduação. O participante E, por sua vez, relatou que realizaria algumas mudanças conforme as demandas emergenciais.

A partir das respostas desses profissionais, é possível perceber que eles buscam conhecimento, o que demonstra a necessidade de que atividades de formação sejam ofertadas a eles, bem como investimentos na sua atuação. Neste cenário, Silva, Oliveira e Kamimura (2014) afirmam que uma capacitação adequada possibilitará o empoderamento do profissional para intervir nos problemas de saúde da população, com um saber e uma prática mais articuladas para atender à complexidade da demanda de quem sofre e procura ajuda. 
No entanto, apesar da reconhecida importância, ainda há poucos investimentos em capacitação, cursos e pós-graduação. Mesmo que existam muitos avanços nesta área, e que as iniciativas possam ser elogiadas, caminhar até a mudança e a aceitação do novo paradigma ainda exigirá capacitação técnica, muita vontade política, além de esforços de todos os envolvidos nesse processo (SILVA; OLIVEIRA; KAMIMURA, 2014).

\title{
Considerações finais
}

Diante dos resultados obtidos no estudo, é possível inferir que os PEF que atuam na $10^{\text {a }}$ CRS do estado do Rio Grande do Sul não têm uma formação inicial acadêmica sobre saúde mental, mas apresentam experiências na atuação nos CAPS ou em serviços relacionados e demonstram satisfação na sua atuação profissional.

Quanto às atividades desenvolvidas com os usuários, concluímos que a atuação prática dos PEF é diversificada, contendo inúmeras propostas de intervenção em diferentes espaços, que vão desde atividades recreativas até aquelas mais voltadas à academia.

Aponta-se que mudanças são necessárias, iniciando pelos cursos de graduação em Educação Física, que devem incluir na formação profissional conteúdos e competências atreladas à atuação no campo da saúde mental, além do investimento em capacitação, de modo a ampliar o conhecimento dos profissionais e contribuir para a melhora do atendimento aos usuários, tornando este um atendimento mais humanizado, integral e pautado nos princípios da reforma psiquiátrica.

Sugerem-se estudos mais profundos sobre a atuação dos PEF nos CAPS para que seja possível aplicar o potencial desta área nos cuidados biopsicossociais. Tais ações poderiam atingir as autoridades responsáveis, dando maiores condições aos profissionais trabalhadores nos CAPS.

\section{PHYSICAL EDUCATION AND MENTAL HEALTH: PROFILE OF THE PROFES- SIONALS AND ACTIVITIES DEVELOPED IN THE MENTAL HEALTH SERVICES}

\begin{abstract}
The objective of the study was to characterize the profile and activities developed by Physical Education Professionals (PEF) in the Mental Health Services of the municipalities that make up the 10th Regional Health Coordination -RS. The data collection was done through a questionnaire composed of open and closed questions. The sample consisted of 6 PEF. The results indicate that PEF did not receive any training related to mental health. The work of the PEF involves varied practices such as sports activities, recreation, taught in the premises of the Mental Health Servicesand in public spaces. In light of the above, it is suggested to include discussions about the subject in undergraduate courses and investment in training for professionals in the area.
\end{abstract}

Keywords: Physical Education. Mental Health Services. Professional Practice.

\section{EDUCACIÓN FÍSICA Y SALUD MENTAL: PERFIL DE LOS PROFESIONALES Y ACTIVIDADES DESARROLLADAS EN LOS CENTROS DE ATENCIÓN PSICO- SOCIAL}

\section{Resumen}

El objetivo del estudio fue caracterizar el perfil y las actividades desarrolladas por los Profesionales de Educación Física (PEF) en los Centros de Atención Psicosocial (CAPS) de los ayuntamientos que componen la $10^{a}$ Coordenadoria Regional de Salud - RS. La recopilación 
de datos se dio a través de un cuestionario compuesto de preguntas abiertas y cerradas. La muestra se compuso de 6 PEF. Los resultados indican que los PEF no recibieron durante su formación inicial ninguna formación relacionada a la salud mental. La actuación de los PEF involucra prácticas variadas como actividades deportivas y recreativas, ofrecidas en las dependencias de los CAPS y en espacios públicos. Frente a lo que se expone, se sugiere la introducción de discusiones sobre el tema en los cursos de graduación y en la capacitación para los profesionales del área.

Palabras clave: Educación física. Servicios de Salud Mental. Práctica Profesional.

\section{Referências}

ABIB, L. T. et al. Práticas corporais em cena na saúde mental: potencialidades de uma oficina de futebol em um Centro de Atenção Psicossocial de Porto Alegre. Pensar a Prática, Goiânia, v. 13, n. 2, p. 1-15, maio/ago. 2010.

ABUHAB, D. et al. O trabalho em equipe multiprofissional no CAPS III: um desafio. Revista Gaúcha de Enfermagem, v. 26, n. 3, p. 369, 2005.

BABINSKI T; HIRDES A. Reabilitação psicossocial: a perspectiva de profissionais de centros de atenção psicossocial do Rio Grande do Sul. Texto Contexto Enferm. 2004, outdez. v. 13 , n. 4, 568-76.

BARDIN, L. Análise de conteúdo. São Paulo, Edições 70, 2011.

BRASIL. Ministério da Saúde. Secretaria de Atenção à Saúde. Departamento de Atenção Básica. Política Nacional de Atenção Básica. Brasília: Ministério da Saúde; 2006.

BRASIL. Lei $\mathrm{n}^{\circ} 10.216$, de 6 de abril de 2001. Dispõe sobre a proteção e os direitos das pessoas portadoras de transtornos mentais e redireciona o modelo assistencial em saúde mental. Brasília, DF.

BRASIL. Ministério da Saúde. Portaria GM no 336, de 19 de fevereiro de 2002.

BRASIL. Ministério da Saúde. Portaria SNAS nº 224, de 29 de janeiro de 1992.

BRASIL. Ministério da Saúde. Secretaria de Atenção à Saúde. DAPE. Coordenação Geral de Saúde Mental. Reforma psiquiátrica e política de saúde mental no Brasil. Brasília: Ministério da Saúde, 2005.

CECCIM, R. B; BILIBIO, L. F. Singularidades da educação física na saúde: desafios à educação de seus profissionais e ao matriciamento interprofissional. In: FRAGA, Alex Branco; WACHS, Felipe. (Org.). Educação física e saúde coletiva: políticas de formação e perspectivas de intervenção. Porto Alegre: UFRGS, 2007. p. 47-62.

CIRQUEIRA, M. V. B. Reflexões acerca da cultura corporal no espaço de atenção à saúde mental. In: RABELO, I. V.; TAVARES, R. C; FARIA, Y. R. A. de (Org.). Olhares experiências de CAPS: Centro de Atenção Psicossocial. Goiânia: Kelps, 2009. p. 53-66. 
CORREIA, R. F. A atividade física e o dependente químico em recuperação. Rev. Ciên. Saúde, v. 2, n. 3, p. 12-15, 2002.

DUARTE, M. L. C; KANTORSKI, L. P. Avaliação da atenção prestada aos familiares em um centro de atenção psicossocial. Rev. Bras. Enferm., v. 64, n. 1, p. 47-52, 2011.

FERREIRA, L. A. S. O trabalho da Educação Física na composição de equipe de saúde mental especializada em álcool e outras drogas. 2013. 109 f. Dissertação (Mestrado em Ciências do Movimento Humano) - Escola de Educação Física, UFRGS, Porto Alegre, 2013.

FURTADO, R. P. et al. O trabalho do professor de Educação Física no CAPS: aproximações iniciais. Movimento, Porto Alegre, v. 21, n. 1, p. 41-52, jan./mar. 2015.

MACHADO, G. J. A atuação do professor de educação física nos centros de atenção psicossocial álcool e drogas da grande Vitória-ES. 2015. 142 f. Dissertação (Mestrado em Educação Física) - Centro de Educação Física e Desporto, UFES, Vitória, 2015.

MACHADO, M. F. A. S. et al. Integralidade, formação de saúde, educação em saúde e as propostas do SUS: uma revisão conceitual. Ciência \& Saúde Coletiva, 2007, v. 12, n. 2, p. $335-342$.

MENEZES, L. E. C. A inserção da Educação Física na equipe multiprofisssional do CAPSi Casa Melodia em Porto Alegre. 2010. 35 f. Monografia (Graduação) - Escola de Educação Física, UFRGS, Porto Alegre, 2010.

MIRANDA, E. D; FREIRE, L. A; OLIVEIRA, A. R. C. Os desafios da Educação Física no Centro de Atenção Psicossocial de Coari (AM). Sau. \&Transf. Soc., Florianópolis, v.1, n.2, p.163-169, 2011.

OLIVEIRA, C. S. et al. O profissional de Educação Física e sua atuação na saúde publica. EFDeportes.com, Buenos Aires, v. 15, n. 153, p. 1, fev. 2011.

OLIVEIRA, F; SILVA, A. Enfermagem em saúde mental no contexto da reabilitação psicossocial e da interdisciplinaridade. Rev. Bras. Enferm., v. 53, n. 4, p. 584-592, out-dez. 2000 .

PIMENTA, E. S; ROMAGNOLI, R. C. A relação com as famílias no tratamento dos portadores de transtorno mental realizado no Centro de Atenção Psicossocial. Pesquisas e Práticas Psicossociais, São João Del Rei, v. 3, n.1, p. 75-84, ago. 2008.

SANTOS, F. T. et al. Atividade física e saúde mental: relato de vivência. Revista Eletrônica Sistemas \& Gestão, Florianópolis, ano 8, n. 12, p. 36-43, 2011.

SARAIVA, S. L; CREMONESE, E. Implantação do modelo de apoio matricial em saúde mental no município de Florianópolis. Brasília: Ministério da Saúde, 2008.

SEVERO, A. K, DIMENSTEIN, M. Rede e intersetorialidade na atenção psicossocial: contextualizando o papel do ambulatório de saúde mental. Psicologia: Ciência e Profissão,v. 31, n. 3, p. 640-655. 
SILVA, S. P; OLIVEIRA, A. L; KAMIMURA, Q. P. Capacitação em saúde mental: entre a realidade e as ofertas do Ministério da Saúde. Revista Eletrônica Sistemas \& Gestão, v. 9, n. 3, p. 406-416, 2014.

TAVARES, C. M. M. A educação permanente da equipe de enfermagem para o cuidado nos serviços de saúde mental. Texto Contexto Enferm, Florianópolis, v. 15, n. 2, p. 287-295, , abr-jun.,2006.

TAVARES, C. M. M. A interdisciplinaridade como requisito para a formação da enfermeira psiquiátrica na perspectiva da atenção psicossocial. Texto Contexto Enferm, v. 14, n. 3, p. 403-410, 2004.

TENÓRIO, F. A Reforma Psiquiátrica Brasileira, da década de 1980 aos dias atuais: história e conceito. História, Ciências, Saúde, Manguinhos, v. 9, n.1, p.25-59, jan./abr. 2002.

WACHS, F. Educação Física e saúde mental: uma prática de cuidado emergente em Centros de Atenção Psicossocial (CAPS). 2008. 147 f. Dissertação (Mestrado em Ciências do Movimento Humano) - Escola de Educação Física, UFRGS, Porto Alegre, 2008.

WEINBERG R. S; GOULD, D. Fundamentos da psicologia do esporte e do exercício. Porto Alegre: Artmed, 2001.

Recebido em: 03/02/2017

Revisado em: 21/06/2017

Aprovado em: 21/06/2017

Endereço para correspondência:

tati_mcs@hotmail.com

Tatiane Motta da Costa e Silva

Universidade Federal do Pampa

BR 472 - Km 592 - Caixa Postal 118

Salso de Baixo

97508000 - Uruguaiana, RS - Brasil 